\title{
The Assouad spectrum of random self-affine carpets
}

\author{
JONATHAN M. FRASER $\dagger$ and SASCHA TROSCHEIT $\ddagger$ \\ $\dagger$ Mathematical Institute, University of St Andrews, North Haugh, St Andrews, \\ Fife, KY16 9SS, UK \\ (e-mail: jmf32@st-andrews.ac.uk) \\ $\ddagger$ Faculty of Mathematics, University of Vienna, Oskar-Morgenstern-Platz 1, \\ 1090 Vienna, Austria \\ (e-mail: sascha.troscheit@univie.ac.at)
}

(Received 13 June 2018 and accepted in revised form 14 July 2020)

\begin{abstract}
We derive the almost sure Assouad spectrum and quasi-Assouad dimension of one-variable random self-affine Bedford-McMullen carpets. Previous work has revealed that the (related) Assouad dimension is not sufficiently sensitive to distinguish between subtle changes in the random model, since it tends to be almost surely 'as large as possible' (a deterministic quantity). This has been verified in conformal and non-conformal settings. In the conformal setting, the Assouad spectrum and quasi-Assouad dimension behave rather differently, tending to almost surely coincide with the upper box dimension. Here we investigate the non-conformal setting and find that the Assouad spectrum and quasi-Assouad dimension generally do not coincide with the box dimension or Assouad dimension. We provide examples highlighting the subtle differences between these notions. Our proofs combine deterministic covering techniques with suitably adapted Chernoff estimates and Borel-Cantelli-type arguments.
\end{abstract}

Key words: Assouad spectrum, quasi-Assouad dimension, random self-affine carpet 2020 Mathematics Subject Classification: 28A80 (Primary); 37C45 (Secondary)

\section{Assouad spectrum and quasi-Assouad dimension}

The Assouad dimension is an important notion of dimension designed to capture extreme local scaling properties of a given metric space. Its distance from the upper box dimension, which measures average global scaling, can be interpreted as a quantifiable measure of inhomogeneity. Motivated by this idea, Fraser and Yu introduced the Assouad spectrum, which is designed to interpolate between the upper box dimension and the Assouad dimension and thus reveal more precise geometric information about the set; see [6]. Here we recall the basic definitions and, for concreteness, we consider non-empty compact 
sets $F \subseteq \mathbb{R}^{d}$, although the general theory extends beyond this setting. For a bounded set $E \subseteq \mathbb{R}^{d}$ and a scale $r>0$ we let $N(E, r)$ be the minimum number of sets of diameter $r$ required to cover $E$. The Assouad dimension of $F$ is defined by

$$
\begin{array}{r}
\operatorname{dim}_{\mathrm{A}} F=\inf \{\alpha:(\exists C>0)(\forall 0<r<R<1) \\
\left.(\forall x \in F) N(B(x, R) \cap F, r) \leqslant C\left(\frac{R}{r}\right)^{\alpha}\right\} .
\end{array}
$$

The Assouad spectrum is the function defined by

$$
\begin{aligned}
\theta \mapsto \operatorname{dim}_{\mathrm{A}}^{\theta} F=\inf \{\alpha:(\exists C>0)(\forall 0<R<1) \\
\left.\quad(\forall x \in F) N\left(B(x, R) \cap F, R^{1 / \theta}\right) \leqslant C\left(\frac{R}{R^{1 / \theta}}\right)^{\alpha}\right\},
\end{aligned}
$$

where $\theta$ varies over $(0,1)$. The related quasi-Assouad dimension is defined by

$$
\begin{array}{r}
\operatorname{dim}_{\mathrm{qA}} F=\lim _{\theta \rightarrow 1} \inf \left\{\alpha:(\exists C>0)\left(\forall 0<r \leqslant R^{1 / \theta} \leqslant R<1\right)\right. \\
\left.(\forall x \in F) N(B(x, R) \cap F, r) \leqslant C\left(\frac{R}{r}\right)^{\alpha}\right\}
\end{array}
$$

and the upper box dimension is defined by

$$
\overline{\operatorname{dim}}_{\mathrm{B}} F=\inf \left\{\alpha:(\exists C)(\forall 0<R<1) N(F, r) \leqslant C\left(\frac{1}{r}\right)^{\alpha}\right\} .
$$

These dimensions are all related, but their relative differences can be subtle. We summarize some important facts to close this section. For any $\theta \in(0,1)$, we have

$$
\overline{\operatorname{dim}}_{\mathrm{B}} F \leqslant \operatorname{dim}_{\mathrm{A}}^{\theta} F \leqslant \operatorname{dim}_{\mathrm{qA}} F \leqslant \operatorname{dim}_{\mathrm{A}} F
$$

and any of these inequalities can be strict. Moreover, the Assouad spectrum is a continuous function of $\theta$ and also satisfies

$$
\operatorname{dim}_{\mathrm{A}}^{\theta} F \leqslant \frac{\overline{\operatorname{dim}}_{\mathrm{B}} F}{1-\theta} .
$$

We also note that for a given $\theta$ it is not necessarily true that the Assouad spectrum is given by the expression after the limit in the definition of the quasi-Assouad dimension: this notion is by definition monotonic in $\theta$, but the spectrum is not necessarily monotonic $[6, \S 8]$. However, it has recently been shown in [4] that $\operatorname{dim}_{\mathrm{qA}} F=\lim _{\theta \rightarrow 1} \operatorname{dim}_{\mathrm{A}}^{\theta} F$ and, combining this with (1.1), we see that the Assouad spectrum necessarily interpolates between the upper box dimension and the quasi-Assouad dimension.

For more information, including basic properties, concerning the upper box dimension, see [2, Chs 2 and 3]. For the Assouad dimension, see [3, 11, 14], for the quasi-Assouad dimension, see [8], and for the Assouad spectrum, see [4, 6, 7]. 


\section{Self-affine carpets: random and deterministic}

In this paper we consider random self-affine carpets. More specifically, one-variable random analogues of the self-affine sets introduced by Bedford and McMullen in the 1980s, where the subdivision is chosen independently and applied uniformly at every iteration of the construction. In the deterministic setting, the box dimensions were computed independently by Bedford and McMullen [1, 13] and the Assouad dimension was computed by Mackay [12]. The Assouad spectrum was computed by Fraser and Yu [7], and these results also demonstrated that the quasi-Assouad and Assouad dimensions coincide by virtue of the spectrum reaching the Assouad dimension. Gatzouras and Lalley [9] first considered stochastically self-affine sets and computed their Hausdorff and box-counting dimensions. This stochastically self-affine model is also known as the random recursive or the $\infty$-variable random model. In the one-variable random setting, the (almost sure) box dimensions were first computed by Gui and $\mathrm{Li}$ [10] for fixed subdivisions and by Troscheit [15] in the most general setting that we are aware of. The (almost sure) Assouad dimension was computed by Fraser, Miao and Troscheit [5]. In this article we compute the quasi-Assouad dimension and the Assouad spectrum in the random setting. Unlike in the deterministic case, we find that the quasi-Assouad dimension and Assouad dimension are usually almost surely distinct. Further, the quasi-Assouad dimension is in general also distinct from the box dimension. This is in stark contrast to the conformal setting, where it was shown that the quasi-Assouad dimension (and thus Assouad spectrum) is almost surely equal to the upper box dimension (and distinct from the Assouad dimension); see [16].

We close this section by describing our model. Let $\Lambda=\{1, \ldots,|\Lambda|\}$ be a finite index set and, for each $i \in \Lambda$, fix integers $n_{i}>m_{i} \geqslant 2$ and divide the unit square [0,1] $]^{2}$ into a uniform $m_{i} \times n_{i}$ grid. For each $i \in \Lambda$, let $\mathcal{I}_{i}$ be a non-empty subset of the set of $m_{i}^{-1} \times n_{i}^{-1}$ rectangles in the grid and let $N_{i}=\left|\mathcal{I}_{i}\right|$. Let $B_{i}$ be the number of distinct columns which contain rectangles from $\mathcal{I}_{i}$, and $C_{i}$ be the maximum number of rectangles in $\mathcal{I}_{i}$ which are contained in a particular column. Note that $1 \leqslant B_{i} \leqslant m_{i}, 1 \leqslant C_{i} \leqslant n_{i}$, and $N_{i} \leqslant B_{i} C_{i}$. For each rectangle $j \in \mathcal{I}_{i}$, let $S_{j}$ be the unique orientation-preserving affine map which maps the unit square $[0,1]^{2}$ onto the rectangle $j$.

Let $\Omega=\Lambda^{\mathbb{N}}$ and, for each $\omega=\left(\omega_{1}, \omega_{2}, \ldots\right) \in \Omega$, we are interested in the corresponding attractor

$$
F_{\omega}=\bigcap_{k \geqslant 1} \bigcup_{j_{1} \in \mathcal{I}_{\omega_{1}}, \ldots, j_{k} \in \mathcal{I}_{\omega_{k}}} S_{j_{1}} \circ \cdots \circ S_{j_{k}}\left([0,1]^{2}\right) .
$$

By randomly choosing $\omega \in \Omega$, we randomly choose an attractor $F_{\omega}$ and we wish to make statements about the generic nature of $F_{\omega}$. For this, we need a measure on $\Omega$. Let $\left\{p_{i}\right\}_{i \in \Lambda}$ be a set of probability weights, that is, for each $i \in \Lambda, 0<p_{i}<1$ and $\sum_{i \in \Lambda} p_{i}=1$. We extend these basic probabilities to a Borel measure $\mathbb{P}$ on $\Omega$ in the natural way, which can be expressed as the infinite-product measure

$$
\mathbb{P}=\prod_{k \in \mathbb{N}} \sum_{i \in \Lambda} p_{i} \delta_{i}
$$

where $\Omega$ is endowed with the product topology and $\delta_{i}$ is a unit mass concentrated at $i \in \Lambda$. Note that the deterministic model can be recovered if $|\Lambda|=1$, that is, there is only one 'pattern' available, which is therefore chosen at every stage in the process. In this case, the 
deterministic attractor is the unique non-empty set $F \subseteq[0,1]^{2}$ satisfying

$$
F=\bigcup_{j \in \mathcal{I}_{1}} S_{j}(F)
$$

\section{Results}

Our main result is an explicit formula which gives the Assouad spectrum of our random self-affine sets almost surely.

THEOREM 3.1. For $\mathbb{P}$ almost all $\omega \in \Omega$, we have

$$
\operatorname{dim}_{\mathrm{A}}^{\theta} F_{\omega}=\left\{\begin{array}{lc}
\frac{1}{1-\theta}\left(\frac{\sum_{i} p_{i} \log \left(B_{i} C_{i}^{\theta} N_{i}^{-\theta}\right)}{\sum_{i} p_{i} \log m_{i}}+\frac{\sum_{i} p_{i} \log \left(N_{i} B_{i}^{-1} C_{i}^{-\theta}\right)}{\sum_{i} p_{i} \log n_{i}}\right) \\
\frac{\sum_{i} p_{i} \log B_{i}}{\sum_{i} p_{i} \log m_{i}}+\frac{\sum_{i} p_{i} \log C_{i}}{\sum_{i} p_{i} \log n_{i}} & \text { for } 0<\theta \leqslant \frac{\sum_{i} p_{i} \log m_{i}}{\sum_{i} p_{i} \log n_{i}}, \\
& \text { for } \frac{\sum_{i} p_{i} \log m_{i}}{\sum_{i} p_{i} \log n_{i}}<\theta<1,
\end{array}\right.
$$

where $F_{\omega}$ is the one-variable random Bedford-McMullen carpet associated with $\omega \in \Omega$.

As an immediate consequence of Theorem 3.1, we obtain a formula for the quasi-Assouad dimension which holds almost surely.

Corollary 3.2. For $\mathbb{P}$ almost all $\omega \in \Omega$, we have

$$
\operatorname{dim}_{\mathrm{qA}} F_{\omega}=\frac{\sum_{i} p_{i} \log B_{i}}{\sum_{i} p_{i} \log m_{i}}+\frac{\sum_{i} p_{i} \log C_{i}}{\sum_{i} p_{i} \log n_{i}},
$$

where $F_{\omega}$ is the one-variable random Bedford-McMullen carpet associated with $\omega \in \Omega$.

Proof. This follows immediately from Theorem 3.1 and the fact that $\operatorname{dim}_{\mathrm{A}}^{\theta} E \rightarrow \operatorname{dim}_{\mathrm{qA}} E$ as $\theta \rightarrow 1$ for any set $E \subseteq \mathbb{R}^{d}$; see [4, Corollary 2.2].

Note that the result in [5] states that for $\mathbb{P}$ almost all $\omega \in \Omega$, we have

$$
\operatorname{dim}_{\mathrm{A}} F_{\omega}=\max _{i \in \Lambda} \frac{\log B_{i}}{\log m_{i}}+\max _{i \in \Lambda} \frac{\log C_{i}}{\log n_{i}} .
$$

Therefore, Corollary 3.2 demonstrates the striking difference between the Assouad and quasi-Assouad dimensions in the random setting. In particular, the almost sure value of the Assouad dimension does not depend on the weights $\left\{p_{i}\right\}_{i \in \Lambda}$, whereas the quasi-Assouad dimension depends heavily on them. The almost sure value of the Assouad dimension is also extremal in the sense that it is the maximum over all realizations $\omega \in \Omega$, whereas the quasi-Assouad dimension is an average. Recall that the quasi-Assouad and Assouad dimensions always coincide for deterministic self-affine carpets; see [7].

It is worth noting that the Assouad dimension of the random attractor is bounded below by the maximal Assouad dimension of the constituent deterministic attractors, whereas the quasi-Assouad dimension is bounded above by the dimension of the individual attractors. 


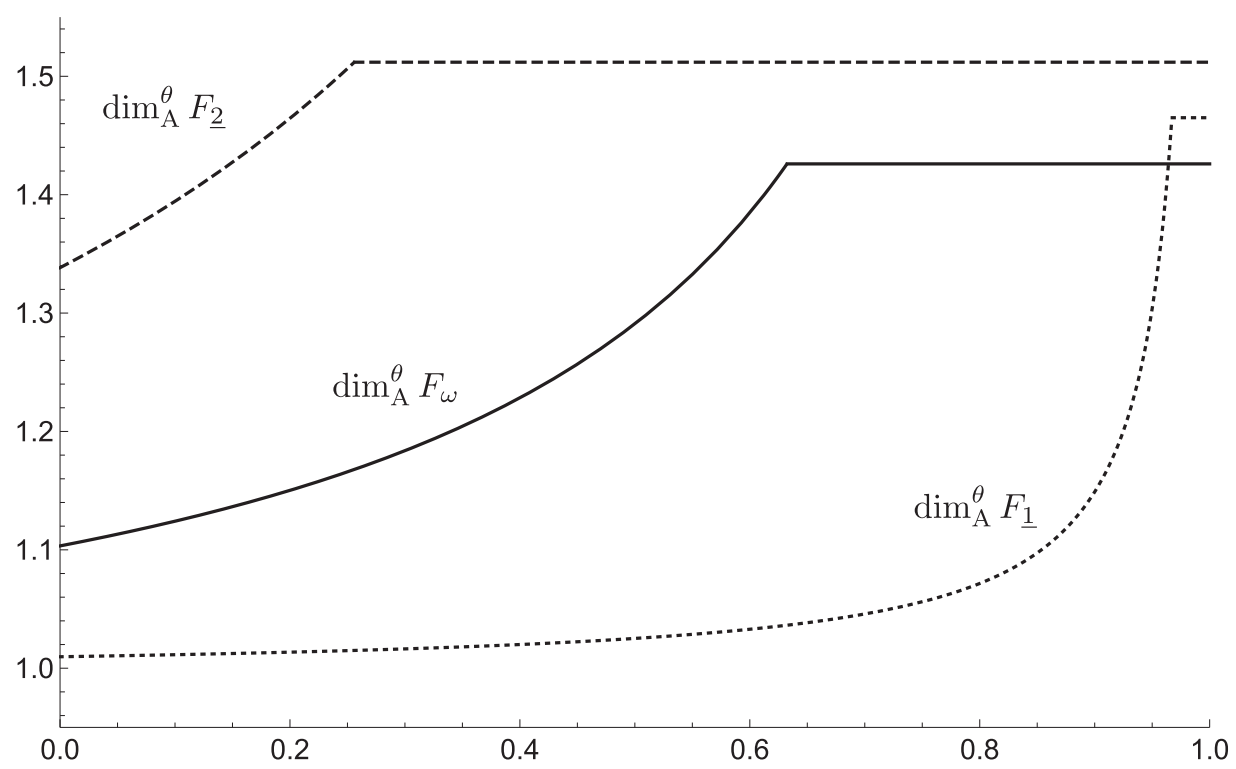

FIGURE 1. The Assouad spectra of the sets in the example of $\$ 3.1$. The deterministic spectra are shown in dashed lines and the almost sure spectrum in the random case is given by a solid line

That is, letting $\underline{i}=(i, i, i, \ldots)$,

$$
\operatorname{dim}_{\mathrm{A}} F_{\omega} \geqslant \max _{i \in \Lambda} \operatorname{dim}_{\mathrm{A}} F_{\underline{i}} \text { (a.s.) and } \operatorname{dim}_{\mathrm{qA}} F_{\omega} \leqslant \max _{i \in \Lambda} \operatorname{dim}_{\mathrm{qA}} F_{\underline{i}} \text { (a.s.). }
$$

Typically these inequalities are strict and it is further possible that $\operatorname{dim}_{\mathrm{qA}} F_{\omega}<$ $\min _{i \in \Lambda} \operatorname{dim}_{\mathrm{qA}} F_{i}$ almost surely; see Figure 1 and the example in Section 3.2.

Finally, note that the almost sure values of the Assouad and quasi-Assouad dimensions coincide if and only if there exist $\alpha, \beta \in(0,1]$ such that for all $i \in \Lambda$, we have $\left(\log B_{i}\right) /\left(\log m_{i}\right)=\alpha$ and $\left(\log C_{i}\right) /\left(\log n_{i}\right)=\beta$. This follows by considering 'weighted mediants'. In particular, the two terms giving the quasi-Assouad dimension are weighted mediants of the fractions $\left(\log B_{i}\right) /\left(\log m_{i}\right)$ and $\left(\log C_{i}\right) /\left(\log n_{i}\right)$, respectively. It is well known that weighted mediants are equal to the maximum if and only if all the fractions coincide. In particular, coincidence of all of the deterministic Assouad (and quasi-Assouad) dimensions is not sufficient to ensure almost sure coincidence of the Assouad and quasi-Assouad dimensions in the random case.

Simple algebraic manipulation yields the following random analogue of [7, Corollary 3.5]. In particular, the random variable $\operatorname{dim}_{\mathrm{A}}^{\theta} F_{\omega}$ can be expressed in terms of the random variables $\overline{\operatorname{dim}}_{\mathrm{B}} F_{\omega}$ and $\operatorname{dim}_{\mathrm{qA}} F_{\omega}$.

COROllary 3.3. For $\mathbb{P}$ almost all $\omega \in \Omega$, we have

$$
\begin{aligned}
\operatorname{dim}_{\mathrm{A}}^{\theta} F_{\omega} & \min \left\{\frac{\overline{\operatorname{dim}}_{\mathrm{B}} F_{\omega}-\theta\left(\operatorname{dim}_{\mathrm{qA}} F_{\omega}-\left(\operatorname{dim}_{\mathrm{qA}} F_{\omega}-\overline{\operatorname{dim}}_{\mathrm{B}} F_{\omega}\right)\left(\sum_{i} p_{i} \log n_{i}\right) /\left(\sum_{i} p_{i} \log m_{i}\right)\right)}{1-\theta},\right. \\
& \left.\operatorname{dim}_{\mathrm{qA}} F\right\},
\end{aligned}
$$

where $F_{\omega}$ is the one-variable random Bedford-McMullen carpet associated with $\omega \in \Omega$. 


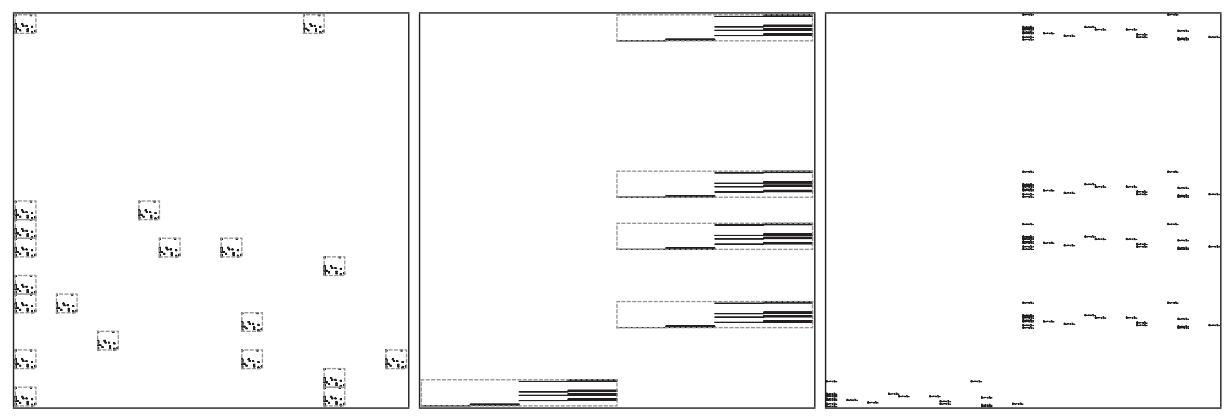

FIGURE 2. The attractors $F_{1}, F_{2}$, and $F_{\omega}$ for $\omega=(2,1,1,2,1, \ldots)$ as used in the example in $\S 3.1$

Note that [7, Corollary 3.5] is formulated using the Assouad dimension instead of the quasi-Assouad dimension (although they are equal in the deterministic case). Our result shows that the quasi-Assouad dimension is really the 'correct' notion to use here.

3.1. Generic example. For illustrative purposes, we exhibit a representative example and provide pictures of the random and deterministic carpets along with their spectra. Let $\Lambda=\{1,2\}$ and $\mathbb{P}$ be the $1 / 2-1 / 2$ Bernoulli probability measure on $\Omega=\Lambda^{\mathbb{N}}$. That is, we consider two iterated function systems that we choose with equal probability.

The first iterated function system consists of $N_{1}=20$ maps, where the unit square is divided into $m_{1}=19$ by $n_{1}=21$ rectangles. There are $B_{1}=10$ columns containing at least one rectangle and the maximal number of rectangles in a particular column is $C_{1}=8$. For the attractor of this deterministic Bedford-McMullen carpet, we obtain

$$
\begin{aligned}
& \operatorname{dim}_{\mathrm{A}} F_{\underline{1}}=\operatorname{dim}_{\mathrm{qA}} F_{\underline{1}}=\frac{\log 10}{\log 19}+\frac{\log 8}{\log 21} \approx 1.465 \text { and } \\
& \overline{\operatorname{dim}}_{\mathrm{B}} F_{\underline{1}}=\frac{\log 10}{\log 19}+\frac{\log 2}{\log 21} \approx 1.010
\end{aligned}
$$

and the spectrum interpolates between these two values with a phase transition at $\log 19 / \log 21 \approx 0.967$. The spectrum is plotted in Figure 1 and the attractor is shown in Figure 2.

The second iterated function system consists of $N_{2}=5$ maps, where the unit square is divided into $m_{2}=2$ by $n_{2}=15$ rectangles. There are $B_{2}=2$ columns containing at least one rectangle and the maximal number of rectangles in a particular column is $C_{2}=4$. For the attractor of this deterministic Bedford-McMullen carpet, we obtain

$$
\operatorname{dim}_{\mathrm{A}} F_{\underline{2}}=\operatorname{dim}_{\mathrm{qA}} F_{\underline{2}}=1+\frac{\log 4}{\log 15} \approx 1.512 \text { and } \overline{\operatorname{dim}}_{\mathrm{B}} F_{\underline{2}}=1+\frac{\log (5 / 2)}{\log 15} \approx 1.338
$$

and the spectrum interpolates between these two values with a phase transition at $\log 2 / \log 15 \approx 0.256$. The spectrum is plotted in Figure 1 and the attractor is shown in Figure 2. 
Our results now give the following values for almost every $\omega \in \Omega$ :

$$
\begin{gathered}
\operatorname{dim}_{\mathrm{A}} F_{\omega}=1+\frac{\log 8}{\log 21} \approx 1.683, \quad \operatorname{dim}_{\mathrm{qA}} F_{\omega}=\frac{\log 20}{\log 38}+\frac{\log 32}{\log 315} \approx 1.426, \\
\text { and } \overline{\operatorname{dim}}_{\mathrm{B}} F_{\omega}=\frac{\log 20}{\log 38}+\frac{\log 5}{\log 315} \approx 1.103 .
\end{gathered}
$$

We note that in this example the almost sure value of the Assouad dimension exceeds that of the individual attractors, that the almost sure quasi-Assouad dimension is less than the quasi-Assouad dimensions of the individual attractors, and that the phase transition in the spectrum occurs at $\log 38 / \log 315 \approx 0.632$.

3.2. An extremal example. By constructing explicit examples, we demonstrate the following interesting phenomenon, which highlights the subtle difference between the quasi-Assouad and Assouad dimensions. For all $\varepsilon \in(0,1)$, there exist two deterministic self-affine carpets $E, F$ with $\overline{\operatorname{dim}}_{\mathrm{B}} E=\overline{\operatorname{dim}}_{\mathrm{B}} F=\operatorname{dim}_{\mathrm{qA}} E=\operatorname{dim}_{\mathrm{qA}} F=\operatorname{dim}_{\mathrm{A}} E=$ $\operatorname{dim}_{\mathrm{A}} F=1$ such that when one mixes the two constructions by randomizing as above, one finds that almost surely

$$
\operatorname{dim}_{\mathrm{qA}} F_{\omega} \leqslant \varepsilon<2=\operatorname{dim}_{\mathrm{A}} F_{\omega} .
$$

Let $\varepsilon \in(0,1), \Lambda=\{1,2\}, m_{1}=2, n_{1}=n, m_{2}=m$, and $n_{2}=m+1$, where $m, n$ are large integers which will be chosen later depending only on $\varepsilon$. Let $\mathcal{I}_{1}$ consist of both rectangles from a particular row in the first grid and $\mathcal{I}_{2}$ consist of all $m+1$ rectangles in a particular column of the second grid. The deterministic carpets associated with these systems are both unit line segments: a horizontal line in the first case and a vertical line in the second. Therefore, both have all the dimensions we consider being equal to 1 . Let $p_{1}=p_{2}=1 / 2$, although the precise choice of weights is not particularly important. It follows that for $\mathbb{P}$ almost all $\omega \in \Omega$, we have

$$
\begin{aligned}
\operatorname{dim}_{\mathrm{qA}} F_{\omega} & =\frac{(1 / 2) \log 2+(1 / 2) \log 1}{(1 / 2) \log 2+(1 / 2) \log m}+\frac{(1 / 2) \log 1+(1 / 2) \log (m+1)}{(1 / 2) \log n+(1 / 2) \log (m+1)} \\
& =\frac{\log 2}{\log (2 m)}+\frac{\log (m+1)}{\log n(m+1)} .
\end{aligned}
$$

Choose $m$ sufficiently large to ensure that $(\log 2) /(\log (2 m)) \leqslant \varepsilon / 2$ and, now that $m$ is fixed, choose $n$ sufficiently large to ensure that $(\log (m+1)) /(\log n(m+1)) \leqslant \varepsilon / 2$. The main result in [5, Theorem 3.2] gives that for any choice of $m, n \geqslant 2, \operatorname{dim}_{\mathrm{A}} F_{\omega}=2$ almost surely and therefore the desired result follows.

\section{Proofs}

4.1. Approximate squares. In this section we introduce (random) approximate squares, which are a common object in the study of self-affine carpets. Fix $\omega=\left(\omega_{1}, \omega_{2}, \ldots\right) \in \Omega$, $R \in(0,1)$, and let $k_{1}^{\omega}(R)$ and $k_{2}^{\omega}(R)$ be the unique positive integers satisfying

$$
\prod_{l=1}^{k_{1}^{\omega}(R)} m_{\omega_{l}}^{-1} \leqslant R<\prod_{l=1}^{k_{1}^{\omega}(R)-1} m_{\omega_{l}}^{-1}
$$


and

$$
\prod_{l=1}^{k_{2}^{\omega}(R)} n_{\omega_{l}}^{-1} \leqslant R<\prod_{l=1}^{k_{2}^{\omega}(R)-1} n_{\omega_{l}}^{-1}
$$

respectively. Also, let

$$
m_{\max }=\max _{i \in \Lambda} m_{i} \quad \text { and } \quad n_{\max }=\max _{i \in \Lambda} n_{i}
$$

A rectangle $[a, b] \times[c, d] \subseteq[0,1]^{2}$ is called an approximate $R$-square if it is of the form

$$
S\left([0,1]^{2}\right) \cap\left(\pi_{1}\left(T\left([0,1]^{2}\right)\right) \times[0,1]\right),
$$

where $\pi_{1}:(x, y) \mapsto x$ is the projection onto the first coordinate and

$$
S=S_{i_{1}} \circ \cdots \circ S_{i_{2}^{\omega}(R)}
$$

and

$$
T=S_{i_{1}} \circ \cdots \circ S_{i_{1} \omega(R)}
$$

for some common sequence $i_{1}, i_{2}, \ldots$ with $i_{j} \in \mathcal{I}_{\omega_{j}}$ for all $j$. Here we say that $Q$ is associated with the sequence $i_{1}, i_{2}, \ldots$, noting that the entries $i_{1}, i_{2}, \ldots, i_{k_{1}^{\omega}(R)}$ determine $Q$. In particular, the base

$$
b-a=\prod_{i=1}^{k_{1}^{\omega}(R)} m_{\omega_{i}}^{-1} \in\left(m_{\max }^{-1} R, R\right] \quad \text { by }(4.1)
$$

and the height

$$
d-c=\prod_{i=1}^{k_{2}^{\omega}(R)} n_{\omega_{i}}^{-1} \in\left(n_{\max }^{-1} R, R\right] \quad \text { by }(4.2)
$$

and so approximate $R$-squares are indeed approximately squares with base and height uniformly comparable to $R$ and therefore to each other.

4.2. Proof strategy and notation. In order to simplify the exposition of our proofs, we define the following weighted geometric averages of the important parameters:

$$
\bar{N}=\prod_{i \in \Lambda} N_{i}^{p_{i}}, \quad \bar{B}=\prod_{i \in \Lambda} B_{i}^{p_{i}}, \quad \bar{C}=\prod_{i \in \Lambda} C_{i}^{p_{i}}, \quad \bar{m}=\prod_{i \in \Lambda} m_{i}^{p_{i}}, \quad \bar{n}=\prod_{i \in \Lambda} n_{i}^{p_{i}} .
$$

Using this notation, in order to prove our result it is sufficient to prove the following two statements.

(1) For all $\log \bar{m} / \log \bar{n}<\theta<1$, we have that for $\mathbb{P}$ almost all $\omega \in \Omega$,

$$
\operatorname{dim}_{\mathrm{A}}^{\theta} F_{\omega} \leqslant \frac{\log \bar{B}}{\log \bar{m}}+\frac{\log \bar{C}}{\log \bar{n}} .
$$


(2) For all $0<\theta<\log \bar{m} / \log \bar{n}$, we have that for $\mathbb{P}$ almost all $\omega \in \Omega$,

$$
\operatorname{dim}_{\mathrm{A}}^{\theta} F_{\omega}=\frac{1}{1-\theta}\left(\frac{\log \bar{B}}{\log \bar{m}}+\frac{\log \bar{N} / \bar{B}}{\log \bar{n}}\right)-\frac{\theta}{1-\theta}\left(\frac{\log (\bar{N} / \bar{C})}{\log \bar{m}}+\frac{\log \bar{C}}{\log \bar{n}}\right) .
$$

To see why this is sufficient, first note that since the Assouad spectrum is a continuous function in $\theta$, see [6, Corollary 3.5], it is determined by its values on a countable dense set and so the above statements imply the a priori stronger statements that for $\mathbb{P}$ almost all $\omega \in \Omega$, we have the given estimates for all $\theta$. Secondly, since the Assouad spectrum necessarily approaches the quasi-Assouad dimension as $\theta \rightarrow 1$, (1) demonstrates that the quasi-Assouad dimension is at most

$$
\frac{\log \bar{B}}{\log \bar{m}}+\frac{\log \bar{C}}{\log \bar{n}}
$$

and, since (2) demonstrates that the Assouad spectrum attains this value at $\theta=$ $\log \bar{m} / \log \bar{n}$, it follows from [6, Corollary 3.6] that it is constant in the interval $[\log \bar{m} / \log \bar{n}, 1)$. Technically speaking, [6, Corollary 3.6] proved that if the Assouad spectrum is equal to the Assouad dimension at some $\theta^{\prime} \in(0,1)$, then it is constant in the interval $\left[\theta^{\prime}, 1\right)$, but the same proof allows one to replace the Assouad dimension with the quasi-Assouad dimension in this statement.

Finally, note that to establish estimates for $\operatorname{dim}_{\mathrm{A}}^{\theta} F_{\omega}$, it suffices to replace balls of radius $R$ with approximate $R$-squares in the definition. That is, to estimate $N\left(Q \cap F_{\omega}, R^{1 / \theta}\right)$, where $Q$ is associated to $i_{1}, i_{2}, \ldots$ with $i_{j} \in \mathcal{I}_{\omega_{j}}$ for all $j$ instead of $N\left(B(x, R) \cap F_{\omega}, R^{1 / \theta}\right)$ for $x \in F_{\omega}$. This is because balls and approximate squares are comparable and one can pass covering estimates concerning one to covering estimates concerning the other up to constant factors. This duality is standard and we do not go into the details.

4.3. Covering estimates. Let $\omega \in \Omega, \theta \in(0,1), R \in(0,1)$, and $Q$ be an approximate $R$-square associated with the sequence $i_{1}, i_{2}, \ldots$ with $i_{j} \in \mathcal{I}_{\omega_{j}}$ for all $j$. In what follows we describe sets of the form $S_{j_{1}} \circ \cdots \circ S_{j_{l}}\left(F_{\omega}\right)$ as level-l cylinders and level- $(l+1)$ cylinders lying inside a particular level- $l$ cylinder will be referred to as children. Moreover, iteration will refer to moving attention from a particular cylinder, or collection of cylinders, to the cylinders at the next level.

We wish to estimate $N\left(Q \cap F_{\omega}, R^{1 / \theta}\right)$ and to do this we decompose $Q \cap F_{\omega}$ into cylinders at level $k_{2}^{\omega}\left(R^{1 / \theta}\right)$ and cover each cylinder independently. Therefore, we first need to count how many level- $k_{2}^{\omega}\left(R^{1 / \theta}\right)$ cylinders lie inside $Q$. There are two cases, which we describe separately.

Case $(i): k_{1}^{\omega}(R)<k_{2}^{\omega}\left(R^{1 / \theta}\right)$. We start by noting that $Q$ lies inside a (unique) level- $k_{2}^{\omega}(R)$ cylinder. As we move to the next level only the children of this cylinder lying in a particular 'column' will also intersect $Q$. Iterating inside cylinders intersecting $Q$ until level $k_{1}^{\omega}(R)$ yields a decomposition of $Q$ into several cylinders arranged in a single column each of which has base the same length as that of $Q$. The number of these cylinders is at most

$$
\prod_{l=k_{2}^{\omega}(R)+1}^{k_{1}^{\omega}(R)} C_{\omega_{l}}
$$


since each iteration from the $(l-1)$ th level to the $l$ th multiplies the number of cylinders intersecting $Q$ at the previous level by the number of rectangles in a particular column of an $\mathcal{I}_{\omega_{l}}$ system, which is, in particular, bounded above by $C_{\omega_{l}}$. The situation is simpler from this point on. We continue to iterate inside each of the level- $k_{1}^{\omega}(R)$ cylinders until level $k_{2}^{\omega}\left(R^{1 / \theta}\right)$, but this time all of the children remain inside $Q$ at every iteration. Therefore, we find precisely

$$
\prod_{l=k_{1}^{\omega}(R)+1}^{k_{2}^{\omega}\left(R^{1 / \theta}\right)} N_{\omega_{l}}
$$

level- $k_{2}^{\omega}\left(R^{1 / \theta}\right)$ cylinders inside each level- $k_{1}^{\omega}(R)$ cylinder. As mentioned above, we now cover each of these cylinders individually. To do this, we further iterate inside each such cylinder until level $k_{1}^{\omega}\left(R^{1 / \theta}\right)$ and group together cylinders at this level which lie in the same column. This decomposes the level- $k_{1}^{\omega}\left(R^{1 / \theta}\right)$ cylinders into approximate $R^{1 / \theta}$-squares, each of which can be covered by four balls of diameter $R^{1 / \theta}$. Therefore, it only remains to count the number of distinct level- $k_{1}^{\omega}\left(R^{1 / \theta}\right)$ columns inside a level- $k_{2}^{\omega}\left(R^{1 / \theta}\right)$ cylinder. Iterating from the $(l-1)$ th level to the $l$ th level multiplies the number of columns by $B_{\omega_{l}}$ and therefore the number is

$$
\prod_{l=k_{2}^{\omega}\left(R^{1 / \theta}\right)+1}^{k_{1}^{\omega}\left(R^{1 / \theta}\right)} B_{\omega_{l}}
$$

Combining the three counting arguments from above yields

$$
N\left(Q \cap F_{\omega}, R^{1 / \theta}\right) \leqslant 4\left(\prod_{l=k_{2}^{\omega}(R)+1}^{k_{1}^{\omega}(R)} C_{\omega_{l}}\right)\left(\prod_{l=k_{1}^{\omega}(R)+1}^{k_{2}^{\omega}\left(R^{1 / \theta}\right)} N_{\omega_{l}}\right)\left(\prod_{l=k_{2}^{\omega}\left(R^{1 / \theta}\right)+1}^{k_{1}^{\omega}\left(R^{1 / \theta}\right)} B_{\omega_{l}}\right)
$$

Moreover, this estimate is sharp in the sense that we can always find a particular approximate $R$-square $Q$ such that

$$
N\left(Q \cap F_{\omega}, R^{1 / \theta}\right) \geqslant K\left(\prod_{l=k_{2}^{\omega}(R)+1}^{k_{1}^{\omega}(R)} C_{\omega_{l}}\right)\left(\prod_{l=k_{1}^{\omega}(R)+1}^{k_{2}^{\omega}\left(R^{1 / \theta}\right)} N_{\omega_{l}}\right)\left(\prod_{l=k_{2}^{\omega}\left(R^{1 / \theta}\right)+1}^{k_{1}^{\omega}\left(R^{1 / \theta}\right)} B_{\omega_{l}}\right)
$$

for some constant $K>0$ depending on $m_{\max }$ and $n_{\max }$. Such a $Q$ is provided by any approximate $R$-square where $T=S_{i_{1}} \circ \cdots \circ S_{i_{k_{1}(R)}}$ is chosen such that each map $i_{j}$ lies in a maximal column of $\mathcal{I}_{j}$, that is, a column consisting of $C_{j}$ rectangles. Finally, the small constant $K$ in the lower bound appears since a single ball of diameter $R^{1 / \theta}$ can only intersect at most a constant number of the approximate $R^{1 / \theta}$-squares found above and therefore counting approximate $R^{1 / \theta}$-squares is still comparable to counting optimal $R^{1 / \theta}$ covers.

Case $(i i): k_{1}^{\omega}(R) \geqslant k_{2}^{\omega}\left(R^{1 / \theta}\right)$. The distinctive feature of this case is that when one iterates inside the level- $k_{2}^{\omega}(R)$ cylinder containing $Q$, one reaches the situation where the height of the cylinders is roughly $R^{1 / \theta}$ (level $\left.k_{2}^{\omega}\left(R^{1 / \theta}\right)\right)$ before the cylinders lie completely inside $Q$ (level $k_{1}^{\omega}(R)$ ). This means that the middle term in the above product no longer 
appears. The rest of the argument is similar, however, and we end up with

$$
N\left(Q \cap F_{\omega}, R^{1 / \theta}\right) \leqslant 4\left(\prod_{l=k_{2}^{\omega}(R)+1}^{k_{2}^{\omega}\left(R^{1 / \theta}\right)} C_{\omega_{l}}\right)\left(\prod_{l=k_{1}^{\omega}(R)+1}^{k_{1}^{\omega}\left(R^{1 / \theta}\right)} B_{\omega_{l}}\right) .
$$

One subtle feature of this estimate is that we appear to skip from level $k_{2}^{\omega}\left(R^{1 / \theta}\right)$ to level $k_{1}^{\omega}(R)$. This is to avoid over-counting due to the fact that, inside a level- $k_{2}^{\omega}\left(R^{1 / \theta}\right)$ cylinder intersecting $Q$, only a single level- $k_{1}^{\omega}(R)$ column actually lies inside $Q$ and can thus contribute to the covering number. This column comprises several $k_{1}^{\omega}(R)$ cylinders and, since the height of this column is comparable to $R^{1 / \theta}$, to cover this column efficiently one only needs to count the number of level- $k_{1}^{\omega}\left(R^{1 / \theta}\right)$ columns inside a single level- $k_{1}^{\omega}(R)$ cylinder. This gives the second multiplicative term in the estimate, which concerns the terms $B_{\omega l}$.

Once again, this bound is sharp in the sense that there exists an approximate $R$-square $Q$ such that

$$
N\left(Q \cap F_{\omega}, R^{1 / \theta}\right) \geqslant K\left(\prod_{l=k_{2}^{\omega}(R)+1}^{k_{2}^{\omega}\left(R^{1 / \theta}\right)} C_{\omega_{l}}\right)\left(\prod_{l=k_{1}^{\omega}(R)+1}^{k_{1}^{\omega}\left(R^{1 / \theta}\right)} B_{\omega_{l}}\right) .
$$

4.4. Proof of the main theorem. We start our proof with this lemma, which is a simple variant of a Chernoff bound for stopped sums of random variables. We write $\mathbb{P}\{a \geqslant b\}$ to denote $\mathbb{P}(\{\omega \in \Omega: a \geqslant b\})$ and write $\mathbb{E}(\cdot)$ for the expectation of a random variable with respect to $\mathbb{P}$.

LEMMA 4.1. Let $\left\{X_{i}\right\}$ be a sequence of non-negative discrete independent and identically distributed random variables with finite expectation $0<\bar{X}=\mathbb{E}(X)<\infty$. Let $\hat{k} \in \mathbb{N}$ and let $k \leqslant \hat{k}$ be a random variable. Let $\tau>\hat{k}$ be a stopping time with finite expectation. Then, for all $\varepsilon, t>0$,

$$
\mathbb{P}\left\{\sum_{i=k}^{\tau} X_{i} \geqslant(1+\varepsilon)(\tau-k+1) \bar{X}\right\} \leqslant \mathbb{E}\left(\mathbb{E}\left(e^{t(X-(1+\varepsilon) \bar{X})}\right)^{\tau-k}\right)
$$

and

$$
\mathbb{P}\left\{\sum_{i=k}^{\tau} X_{i} \leqslant(1-\varepsilon)(\tau-k+1) \bar{X}\right\} \leqslant \mathbb{E}\left(\mathbb{E}\left(e^{t(X-(1+\varepsilon) \bar{X})}\right)^{\tau-k}\right) .
$$

Further, if $\tau-k \geqslant l$ for some $l \in \mathbb{N}$, then there exists $0<\gamma<1$ not depending on $\tau, k, l$ such that

$$
\mathbb{P}\left\{\sum_{i=k}^{\tau} X_{i} \geqslant(1+\varepsilon)(\tau-k+1) \bar{X}\right\} \leqslant \gamma^{l}
$$

and

$$
\mathbb{P}\left\{\sum_{i=k}^{\tau} X_{i} \leqslant(1-\varepsilon)(\tau-k+1) \bar{X}\right\} \leqslant \gamma^{l}
$$


Proof. In what follows, we write $\left\{\mathcal{F}_{s}\right\}_{s} \geqslant 0$ for the natural filtration of our event space. We prove (4.6) and (4.8). The remaining estimates are proved similarly and we omit the details. We rearrange the left-hand side of (4.6) and multiply by $t>0$ to obtain

$$
\begin{aligned}
\mathbb{P}\left\{\sum_{i=k}^{\tau} X_{i} \geqslant(1+\varepsilon)(\tau-k+1) \bar{X}\right\} & =\mathbb{P}\left\{\sum_{i=k}^{\tau} t\left(X_{i}-(1+\varepsilon) \bar{X}\right) \geqslant 0\right\} \\
& =\mathbb{P}\left\{\exp \left[\sum_{i=k}^{\tau} Y_{i}\right] \geqslant 1\right\}
\end{aligned}
$$

with $Y_{i}=t X_{i}-t(1+\varepsilon) \bar{X}$. Using Markov's inequality and continuing,

$$
\begin{aligned}
& \leqslant \mathbb{E}\left(\exp \left[\sum_{i=k}^{\tau} Y_{i}\right]\right) \\
& =\mathbb{E}\left(\mathbb{E}\left(\exp \left[\sum_{i=k}^{\tau} Y_{i}\right] \mid \mathcal{F}_{\tau-1}\right)\right) \\
& =\mathbb{E}\left(\mathbb{E}\left(\exp Y_{\tau} \mid \mathcal{F}_{\tau-1}\right) \mathbb{E}\left(\exp \left[\sum_{i=k}^{\tau-1} Y_{i}\right] \mid \mathcal{F}_{\tau-1}\right)\right) \\
& =\mathbb{E}\left(\mathbb{E}\left(\exp Y_{0}\right) \mathbb{E}\left(\mathbb{E}\left(\exp \left[\sum_{i=k}^{\tau-1} Y_{i}\right] \mid \mathcal{F}_{\tau-2}\right) \mid \mathcal{F}_{\tau-1}\right)\right) \\
& =\mathbb{E}\left(\mathbb{E}\left(\exp Y_{0}\right)^{2} \mathbb{E}\left(\mathbb{E}\left(\exp \left[\sum_{i=k}^{\tau-2} Y_{i}\right] \mid \mathcal{F}_{\tau-2}\right) \mid \mathcal{F}_{\tau-1}\right)\right) \\
& \vdots \\
& =\mathbb{E}\left(\mathbb{E}\left(\exp Y_{0}\right)^{\tau-k}\right) \\
& =\mathbb{E}\left(\mathbb{E}\left(e^{t(X-(1+\varepsilon) \bar{X})}\right)^{\tau-k}\right),
\end{aligned}
$$

as required.

To prove (4.8), we consider

$$
\gamma_{t}=\mathbb{E}\left(e^{t\left(X_{0}-(1+\varepsilon) \bar{X}\right)}\right)
$$

Since $X$ is discrete, we can differentiate with respect to $t$ for all $t \in \mathbb{R}$ and get

$$
\begin{aligned}
\left.\frac{d}{d t} \mathbb{E}\left(e^{t\left(X_{0}-(1+\varepsilon) \bar{X}\right)}\right)\right|_{t=0} & =\left.\mathbb{E}\left(\frac{d}{d t} e^{t\left(X_{0}-(1+\varepsilon) \bar{X}\right)}\right)\right|_{t=0} \\
& =\left.\mathbb{E}\left(\left(X_{0}-(1+\varepsilon) \bar{X}\right) e^{t\left(X_{0}-(1+\varepsilon) \bar{X}\right)}\right)\right|_{t=0} \\
& =\mathbb{E}\left(X_{0}-(1+\varepsilon) \bar{X}\right)=-\varepsilon \bar{X}<0 .
\end{aligned}
$$

Thus, since $\gamma_{0}=1$, there exists $t>0$ such that $0<\gamma_{t}<1$. Note that $t$ (and thus $\gamma_{t}$ ) does not depend on on $\tau, k, l$ and we can now use (4.6) together with the assumption that $\tau-$ $k \geqslant l$ to obtain (4.8), where $\gamma=\gamma_{t}$. 
Note that from the definitions of $k_{1}^{\omega}(R)$ and $k_{2}^{\omega}(R)$ we can conclude that there exist constants $c_{1}, c_{\theta}>1$ such that for sufficiently small $R$,

$$
k_{1}^{\omega}(R) \geqslant c_{1} k_{2}^{\omega}(R), \quad k_{1}^{\omega}\left(R^{1 / \theta}\right) \geqslant c_{\theta} k_{1}^{\omega}(R), \quad \text { and } \quad k_{2}^{\omega}\left(R^{1 / \theta}\right) \geqslant c_{\theta} k_{2}^{\omega}(R) .
$$

The relationship between $k_{1}^{\omega}(R)$ and $k_{2}^{\omega}\left(R^{1 / \theta}\right)$ is more complicated and depends heavily on $\omega$ and $R$. However, probabilistically we can say more. Let $\varepsilon>0$ and $q \in \mathbb{N}$. Note that, taking logarithms,

$$
\mathbb{P}\left\{\prod_{i=1}^{q} n_{\omega_{i}}^{-1} \leqslant(\bar{n})^{-(1+\varepsilon) q}\right\}=\mathbb{P}\left\{\sum_{i=1}^{q} \log n_{\omega_{i}} \geqslant(1+\varepsilon) q \log \bar{n}\right\}
$$

and therefore, by Lemma 4.1, there exists $0<\gamma<1$ such that

$$
\mathbb{P}\left\{\prod_{i=1}^{q} n_{\omega_{i}}^{-1} \leqslant(\bar{n})^{-(1+\varepsilon) q}\right\} \leqslant \gamma^{q-1} .
$$

Now, summing over $q$, we obtain

$$
\sum_{q=1}^{\infty} \mathbb{P}\left\{\prod_{i=1}^{q} n_{\omega_{i}}^{-1} \leqslant(\bar{n})^{-(1+\varepsilon) q}\right\} \leqslant \sum_{q=1}^{\infty} \gamma^{q-1}<\infty .
$$

Thus, by the Borel-Cantelli lemma, almost surely there are at most finitely many $q$ such that these events occur. We can similarly argue for a lower bound and conclude that for almost all $\omega \in \Omega$, there exists $q_{\omega}$ such that

$$
(\bar{n})^{-(1+\varepsilon) q} \leqslant \prod_{i=1}^{q} n_{\omega_{i}}^{-1} \leqslant(\bar{n})^{-(1-\varepsilon) q}
$$

for all $q \geqslant q_{\omega}$. Analogously,

$$
(\bar{m})^{-(1+\varepsilon) q} \leqslant \prod_{i=1}^{q} m_{\omega_{i}}^{-1} \leqslant(\bar{m})^{-(1-\varepsilon) q}
$$

almost surely for all $q$ large enough. Without loss of generality, we can assume $q_{\omega}$ to be identical for both products. Since $k_{2}^{\omega}(R) \geqslant-c \log R$ for some $c>0$ not depending on $\omega, R$, we see that almost surely there also exists an $R_{\omega}$ such that (4.9) and (4.10) hold for all $q \geqslant k_{2}^{\omega}(R)$, where $0<R \leqslant R_{\omega}$.

Given these bounds, we can determine the probabilistic relationship between $k_{1}^{\omega}(R)$ and $k_{2}^{\omega}\left(R^{1 / \theta}\right)$. Let $R_{\omega}$ be as above. Then, by the definitions of $k_{1}^{\omega}(R)$ and $k_{2}^{\omega}\left(R^{1 / \theta}\right)$, we get, for all $R \leqslant R_{\omega}$,

$$
(\bar{m})^{-(1+\varepsilon) k_{1}^{\omega}(R)} \leqslant \prod_{i=1}^{k_{1}^{\omega}(R)} m_{\omega_{i}}^{-1} \leqslant R<n_{\max }^{\theta}\left(\prod_{i=1}^{k_{2}^{\omega}\left(R^{1 / \theta}\right)} n_{\omega_{i}}^{-1}\right)^{\theta} \leqslant n_{\max }^{\theta}(\bar{n})^{-\theta(1-\varepsilon) k_{2}^{\omega}\left(R^{1 / \theta}\right)}
$$

and, after rearranging,

$$
\frac{k_{1}^{\omega}(R)}{k_{2}^{\omega}\left(R^{1 / \theta}\right)}>\theta \frac{1-\varepsilon}{1+\varepsilon} \frac{\log \bar{n}}{\log \bar{m}}-\theta \frac{\log n_{\max }}{(1+\varepsilon) k_{2}^{\omega}\left(R^{1 / \theta}\right) \log \bar{m}} .
$$


Similarly, by considering the complementary inequalities

$$
\left(\prod_{i=1}^{k_{2}^{\omega}\left(R^{1 / \theta}\right)} n_{\omega_{i}}^{-1}\right)^{\theta} \leqslant R<m_{\max } \prod_{i=1}^{k_{1}^{\omega}(R)} m_{\omega_{i}}^{-1}
$$

we obtain

$$
\frac{k_{1}^{\omega}(R)}{k_{2}^{\omega}\left(R^{1 / \theta}\right)}<\theta \frac{1+\varepsilon}{1-\varepsilon} \frac{\log \bar{n}}{\log \bar{m}}-\frac{\log m_{\max }}{(1-\varepsilon) k_{2}^{\omega}\left(R^{1 / \theta}\right) \log \bar{m}} .
$$

Now $\varepsilon>0$ was arbitrary and the last term in (4.11) and (4.12) vanishes as $R_{\omega}$ decreases. Therefore, for all $\delta>0$ and almost all $\omega \in \Omega$, there exists a sufficiently small $R_{\omega}>0$ such that

$$
(1-\delta) \frac{\theta \log \bar{n}}{\log \bar{m}} \leqslant \frac{k_{1}^{\omega}(R)}{k_{2}^{\omega}\left(R^{1 / \theta}\right)} \leqslant(1+\delta) \frac{\theta \log \bar{n}}{\log \bar{m}}
$$

for all $R<R_{\omega}$. Moreover, using the much simpler relationships derived above, we can assume without loss of generality that $R_{\omega}$ is small enough such that

$$
\begin{gathered}
(1-\delta) \frac{\log \bar{n}}{\log \bar{m}} \leqslant \frac{k_{1}^{\omega}(R)}{k_{2}^{\omega}(R)} \leqslant(1+\delta) \frac{\log \bar{n}}{\log \bar{m}}, \\
(1-\delta) \theta \leqslant \frac{k_{1}^{\omega}(R)}{k_{1}^{\omega}\left(R^{1 / \theta}\right)} \leqslant(1+\delta) \theta \quad \text { and } \quad(1-\delta) \theta \leqslant \frac{k_{2}^{\omega}(R)}{k_{2}^{\omega}\left(R^{1 / \theta}\right)} \leqslant(1+\delta) \theta
\end{gathered}
$$

all hold simultaneously for all $R<R_{\omega}$.

4.4.1. The upper bound for $\theta<\log \bar{m} / \log \bar{n}$. We assume throughout that $R_{\omega}$ is small enough for all inequalities in the previous section to hold simultaneously (almost surely). Also, let $\delta>0$ be small enough such that the inequalities at the end of the previous section are all bounded away from 1 . That is, we choose $\delta>0$ such that $(1+\delta) \theta<1$, $(1-$ $\delta)(\log \bar{n} / \log \bar{m})>1$, and, especially relevant to this section, (4.13) and $\theta<\log \bar{m} / \log \bar{n}$ imply that we can choose $\delta>0$ sufficiently small such that $k_{1}^{\omega}(R)<k_{2}^{\omega}\left(R^{1 / \theta}\right)$ almost surely for all $R<R_{\omega}$.

Let $\varepsilon>0$ and consider the geometric average given by

$$
\left(\bar{C}^{k_{1}^{\omega}(R)-k_{2}^{\omega}(R)} \bar{N}_{2}^{k_{2}^{\omega}\left(R^{1 / \theta}\right)-k_{1}^{\omega}(R)} \bar{B}_{1}^{k_{1}^{\omega}\left(R^{1 / \theta}\right)-k_{2}^{\omega}\left(R^{1 / \theta}\right)}\right)^{1+\varepsilon} .
$$

We want to determine the probability that there exists an approximate $R$-square at a given level such that we need more than the estimate in (4.16) many $R^{1 / \theta}$-squares to cover it. Note that for (4.3) to be larger than (4.16), at least one of the products must exceed the corresponding power of the average. Therefore,

$$
\begin{aligned}
& \mathbb{P}\left\{N\left(Q \cap F_{\omega}, R^{1 / \theta}\right) \geqslant 4\left(\bar{C}^{k_{1}^{\omega}(R)-k_{2}^{\omega}(R)} \bar{N}^{k_{2}^{\omega}\left(R^{1 / \theta}\right)-k_{1}^{\omega}(R)} \bar{B}^{k_{1}^{\omega}\left(R^{1 / \theta}\right)-k_{2}^{\omega}\left(R^{1 / \theta}\right)}\right)^{1+\varepsilon}\right\} \\
& \quad \leqslant \mathbb{P}\left\{\left(\prod_{l=k_{2}^{\omega}(R)+1}^{k_{1}^{\omega}(R)} C_{\omega_{l}}\right) \geqslant \bar{C}^{(1+\varepsilon)\left(k_{1}^{\omega}(R)-k_{2}^{\omega}(R)\right)}\right\}
\end{aligned}
$$




$$
\begin{aligned}
& +\mathbb{P}\left\{\left(\prod_{l=k_{1}^{\omega}(R)+1}^{k_{2}^{\omega}\left(R^{1 / \theta}\right)} N_{\omega_{l}}\right) \geqslant \bar{N}^{(1+\varepsilon)\left(k_{2}^{\omega}\left(R^{1 / \theta}\right)-k_{1}^{\omega}(R)\right)}\right\} \\
& +\mathbb{P}\left\{\left(\prod_{l=k_{2}^{\omega}\left(R^{1 / \theta}\right)+1}^{k_{1}^{\omega}\left(R^{1 / \theta}\right)} B_{\omega_{l}}\right) \geqslant \bar{B}^{(1+\varepsilon)\left(k_{1}^{\omega}\left(R^{1 / \theta}\right)-k_{2}^{\omega}\left(R^{1 / \theta}\right)\right)}\right\} .
\end{aligned}
$$

Let us start by analysing the event involving $C_{\omega_{l}}$. We want to show that the product can only exceed the average behaviour at most finitely many times almost surely. That is, given $q \in \mathbb{N}$, we want to estimate

$$
\mathbb{P}\left\{\prod_{l=k_{2}^{\omega}(R)+1}^{k_{1}^{\omega}(R)} C_{\omega_{l}} \geqslant \bar{C}^{(1+\varepsilon)\left(k_{1}^{\omega}(R)-k_{2}^{\omega}(R)\right)} \text { for some } R \in\left(0, R_{\omega}\right) \text { such that } k_{2}^{\omega}(R)=q\right\} .
$$

Notice that $k_{1}^{\omega}(R)$ is a stopping time and, by (4.14) and our assumption that $R_{\omega}$ and $\delta$ are chosen sufficiently small, $k_{1}^{\omega}(R) \geqslant(1-\delta) \log (\bar{n}) / \log (\bar{m}) k_{2}^{\omega}(R)$ and $c:=(1-$ $\delta) \log (\bar{n}) / \log (\bar{m})-1>0$. Using Lemma 4.1, we can bound (4.18) above by

$$
\mathbb{P}\left\{\underset{\substack{q^{\prime}: \exists R \in\left(0, R_{\omega}\right) \\ k_{2}^{\omega}(R)=q \text { and } k_{1}^{\omega}(R)=q^{\prime}}}{\bigcup_{l=q+1}^{q^{\prime}}}\left\{\sum_{l=1} \log C_{\omega_{l}} \geqslant(1+\varepsilon)\left(q^{\prime}-q\right) \log \bar{C}\right\}\right\} \leqslant L \gamma^{c(q-1)}
$$

for some $0<\gamma<1$, where $L>0$ is a deterministic constant corresponding to the number of possible values for $k_{1}^{\omega}(R)$ given $k_{2}^{\omega}(R)$. Since

$$
\sum_{q=1}^{\infty} L \gamma^{c(q-1)}<\infty
$$

the Borel-Cantelli lemma implies that the product can exceed the average behaviour only finitely many times almost surely. The argument for $N_{\omega_{l}}$ and $B_{\omega_{l}}$ is identical due to the ratios given in (4.13), (4.14), and (4.15). Therefore, there almost surely exists $q$ large enough—and hence $R_{\omega}^{\prime}$ small enough—such that

$$
N\left(Q \cap F_{\omega}, R^{1 / \theta}\right) \leqslant 4\left(\bar{C}^{k_{1}^{\omega}(R)-k_{2}^{\omega}(R)} \bar{N}^{k_{2}^{\omega}\left(R^{1 / \theta}\right)-k_{1}^{\omega}(R)} \bar{B}^{k_{1}^{\omega}\left(R^{1 / \theta}\right)-k_{2}^{\omega}\left(R^{1 / \theta}\right)}\right)^{1+\varepsilon}
$$

for all $0<R<R_{\omega}^{\prime}$. Using (4.13), (4.14), and (4.15) again, we obtain

$$
\begin{gathered}
k_{1}^{\omega}(R)-k_{2}^{\omega}(R) \leqslant\left((1+\delta) \frac{\log \bar{n}}{\log \bar{m}}-1\right) k_{2}^{\omega}(R), \\
k_{2}^{\omega}\left(R^{1 / \theta}\right)-k_{1}^{\omega}(R) \leqslant\left(\frac{1+\delta}{1-\delta} \theta^{-1}-(1+\delta) \frac{\log \bar{n}}{\log \bar{m}}\right) k_{2}^{\omega}(R),
\end{gathered}
$$

and

$$
k_{1}^{\omega}\left(R^{1 / \theta}\right)-k_{2}^{\omega}\left(R^{1 / \theta}\right) \leqslant\left(\frac{1+\delta}{1-\delta} \frac{\log \bar{n}}{\log \bar{m}}-(1-\delta)^{-1}\right) \theta^{-1} k_{2}^{\omega}(R)
$$


Now, using $k_{2}^{\omega}(R) \leqslant-(1+\delta) \log R / \log \bar{n}$, we rearrange

$$
\bar{C}^{k_{1}^{\omega}(R)-k_{2}^{\omega}(R)} \leqslant \bar{C}^{-(1+\delta)^{2} \log R / \log \bar{m}-(-(1+\delta) \log R / \log \bar{n})}=R^{(1-1 / \theta) s_{c}},
$$

where

$$
\begin{aligned}
s_{c}= & (1+\delta)^{2} \frac{\log \bar{C}}{\log \bar{m}} \frac{\theta}{1-\theta}-(1+\delta) \frac{\log \bar{C}}{\log \bar{n}} \frac{\theta}{1-\theta} \\
& \rightarrow s_{C}:=\frac{\theta}{1-\theta}\left(\frac{\log \bar{C}}{\log \bar{m}}-\frac{\log \bar{C}}{\log \bar{n}}\right) \text { as } \delta \rightarrow 0 .
\end{aligned}
$$

We rearrange the other terms similarly to obtain

$$
N\left(Q \cap F_{\omega}, R^{1 / \theta}\right) \leqslant 4 R^{(1-1 / \theta)(1+\varepsilon)\left(s_{c}+s_{n}+s_{b}\right)},
$$

where

$$
\begin{aligned}
s_{n}= & -(1+\delta)^{2} \frac{\log \bar{N}}{\log \bar{m}} \frac{\theta}{1-\theta}+\frac{(1+\delta)^{2}}{1-\delta} \frac{\log \bar{N}}{\log \bar{n}} \frac{1}{1-\theta} \\
& \rightarrow s_{N}:=\frac{1}{1-\theta}\left(\frac{\log \bar{N}}{\log \bar{n}}-\theta \frac{\log \bar{N}}{\log \bar{m}}\right) \text { as } \delta \rightarrow 0
\end{aligned}
$$

and

$$
\begin{aligned}
s_{b}= & -\frac{1+\delta}{1-\delta} \frac{\log \bar{B}}{\log \bar{n}} \frac{1}{1-\theta}+\frac{(1+\delta)^{2}}{1-\delta} \frac{\log \bar{B}}{\log \bar{m}} \frac{1}{1-\theta} \\
& \rightarrow s_{B}:=\frac{1}{1-\theta}\left(\frac{\log \bar{B}}{\log \bar{m}}-\frac{\log \bar{B}}{\log \bar{n}}\right) \text { as } \delta \rightarrow 0
\end{aligned}
$$

For arbitrary $\varepsilon^{\prime}>0$, we may assume that $\delta>0$ is small enough such that $s_{c}+s_{n}+s_{b} \leqslant$ $\left(1+\varepsilon^{\prime}\right)\left(s_{C}+s_{N}+s_{B}\right)$. Note that

$s:=s_{C}+s_{N}+s_{B}=\frac{1}{1-\theta}\left[\left(\frac{\log \bar{B}}{\log \bar{m}}+\frac{\log \bar{N} / \bar{B}}{\log \bar{n}}\right)-\theta\left(\frac{\log \bar{N} / \bar{C}}{\log \bar{m}}+\frac{\log \bar{C}}{\log \bar{n}}\right)\right]$.

We can therefore conclude that, almost surely, every approximate square of length $R<R_{\omega}$ can be covered by fewer than

$$
4 R^{(1-1 / \theta)(1+\varepsilon)\left(1+\varepsilon^{\prime}\right) s}
$$

sets of diameter $R^{1 / \theta}$. Thus, the Assouad spectrum is bounded above by $(1+\varepsilon)\left(1+\varepsilon^{\prime}\right) s$ and, by the arbitrariness of $\varepsilon, \varepsilon^{\prime}$, also by $s$.

4.4.2. The upper bound for $\theta>\log \bar{m} / \log \bar{n}$. The proof for this case follows along the same lines as $\theta<\log \bar{m} / \log \bar{n}$ and we will only sketch their differences. First note that $\theta>\log \bar{m} / \log \bar{n}$ implies the almost sure existence of a small enough $R_{\omega}$ such that

$$
k_{1}^{\omega}(R) \geqslant(1-\delta) \theta \frac{\log \bar{n}}{\log \bar{m}} k_{2}^{\omega}\left(R^{1 / \theta}\right)
$$

for all $R<R_{\omega}$. Again, we assume without loss of generality that $R_{\omega}$ is chosen such that (4.13), (4.14), and (4.15) are satisfied for a given $\delta>0$. We also choose $\delta>0 \mathrm{small}$ enough 
to ensure that

$$
(1-\delta) \theta \frac{\log \bar{n}}{\log \bar{m}}>1 .
$$

Let $\varepsilon>0$ and consider the geometric average

$$
\left(\bar{C}^{k_{2}^{\omega}\left(R^{1 / \theta}\right)-k_{2}^{\omega}(R)} \bar{B}^{k_{1}^{\omega}\left(R^{1 / \theta}\right)-k_{1}^{\omega}(R)}\right)^{1+\varepsilon} .
$$

We compare the upper bound given in (4.5) with the average above and obtain

$$
\begin{aligned}
\mathbb{P}\left\{N\left(Q \cap F_{\omega}, R^{1 / \theta}\right) \geqslant\right. & \left.4\left(\bar{C}_{2}^{k_{2}^{\omega}\left(R^{1 / \theta}\right)-k_{2}^{\omega}(R)} \bar{B}^{k_{1}^{\omega}\left(R^{1 / \theta}\right)-k_{1}^{\omega}(R)}\right)^{1+\varepsilon}\right\} \\
\leqslant & \mathbb{P}\left\{\left(\prod_{l=k_{2}^{\omega}(R)+1}^{k_{2}^{\omega}\left(R^{1 / \theta}\right)} C_{\omega_{l}}\right) \geqslant \bar{C}^{(1+\varepsilon)\left(k_{2}^{\omega}\left(R^{1 / \theta}\right)-k_{2}^{\omega}(R)\right)}\right\} \\
& +\mathbb{P}\left\{\left(\prod_{l=k_{1}^{\omega}(R)+1}^{k_{1}^{\omega}\left(R^{1 / \theta}\right)} B_{\omega_{l}}\right) \geqslant \bar{B}^{(1+\varepsilon)\left(k_{1}^{\omega}\left(R^{1 / \theta}\right)-k_{1}^{\omega}(R)\right)}\right\} .
\end{aligned}
$$

Now, using the same ideas as before, noting that $k_{1}^{\omega}(\cdot)$ and $k_{2}^{\omega}(\cdot)$ are stopping times, we can conclude that for almost every $\omega \in \Omega$ there exists $R_{\omega}$ such that

$$
N\left(Q \cap F_{\omega}, R^{1 / \theta}\right) \leqslant 4\left(\bar{C}^{k_{2}^{\omega}\left(R^{1 / \theta}\right)-k_{2}^{\omega}(R)} \bar{B}^{k_{1}^{\omega}\left(R^{1 / \theta}\right)-k_{1}^{\omega}(R)}\right)^{1+\varepsilon}
$$

for all $R<R_{\omega}$. Using the estimates for $k_{1}^{\omega}\left(R^{1 / \theta}\right) / k_{1}^{\omega}(R)$ and $k_{2}^{\omega}\left(R^{1 / \theta}\right) / k_{2}^{\omega}(R)$ in (4.15), we see that there exists $\varepsilon^{\prime}>0$ such that for sufficiently small $R$,

$$
\left(\bar{C}^{k_{2}^{\omega}\left(R^{1 / \theta}\right)-k_{2}^{\omega}(R)} \bar{B}^{k_{1}^{\omega}\left(R^{1 / \theta}\right)-k_{1}^{\omega}(R)}\right)^{1+\varepsilon} \leqslant R^{(1-1 / \theta)(1+\varepsilon)\left(1+\varepsilon^{\prime}\right) s},
$$

where

$$
s=\frac{\log \bar{B}}{\log \bar{m}}+\frac{\log \bar{C}}{\log \bar{n}} .
$$

As before, this is sufficient to prove that for $\theta>\log \bar{m} / \log \bar{n}$, there almost surely exists $R_{\omega}$ such that all approximate $R$-squares with $R<R_{\omega}$ can be covered by fewer than

$$
4 R^{(1-1 / \theta)(1+\varepsilon)\left(1+\varepsilon^{\prime}\right) s}
$$

sets of diameter $R^{1 / \theta}$. This proves that $\operatorname{dim}_{\mathrm{A}}^{\theta} F_{\omega} \leqslant(1+\varepsilon)\left(1+\varepsilon^{\prime}\right) s$ almost surely and hence, by arbitrariness of $\varepsilon, \varepsilon^{\prime}>0$, that $\operatorname{dim}_{\mathrm{A}}^{\theta} F_{\omega} \leqslant s$ almost surely, as required.

4.4.3. The lower bound for $\theta<\log \bar{m} / \log \bar{n}$. To prove almost sure lower bounds for $\operatorname{dim}_{\mathrm{A}}^{\theta} F_{\omega}$, we need to show that almost surely there exists a sequence $R_{i} \rightarrow 0$ such that for each $i$ there is an approximate $R_{i}$-square which requires at least a certain number of sets of diameter $R_{i}^{1 / \theta}$ to cover it.

Let $\theta<\log \bar{m} / \log \bar{n}$ and, as before, we choose $\delta>0$ small enough such that $k_{1}^{\omega}(R)<$ $k_{2}^{\omega}\left(R^{1 / \theta}\right)$ almost surely for all small enough $R$. Let $\varepsilon>0$ and, given $q \in \mathbb{N}$ and $\omega \in \Omega$, let

$$
R_{q}=\prod_{l=1}^{q} n_{\omega_{l}}^{-1},
$$


noting that $k_{2}^{\omega}\left(R_{q}\right)=q$ and $R_{q} \rightarrow 0$ as $q \rightarrow \infty$. We have

$$
\begin{aligned}
& \mathbb{P}\left\{N\left(Q \cap F_{\omega}, R_{q}^{1 / \theta}\right) \geqslant K\left(\bar{C}^{k_{1}^{\omega}\left(R_{q}\right)-k_{2}^{\omega}\left(R_{q}\right)} \bar{N}^{k_{2}^{\omega}\left(R_{q}^{1 / \theta}\right)-k_{1}^{\omega}\left(R_{q}\right)} \bar{B}_{1}^{k_{1}^{\omega}\left(R_{q}^{1 / \theta}\right)-k_{2}^{\omega}\left(R_{q}^{1 / \theta}\right)}\right)^{1-\varepsilon}\right\} \\
& \geqslant 1-\mathbb{P}\left\{\prod_{l=k_{2}^{\omega}\left(R_{q}\right)+1}^{k_{1}^{\omega}\left(R_{q}\right)} C_{\omega_{l}} \leqslant \bar{C}^{(1-\varepsilon)\left(k_{1}^{\omega}\left(R_{q}\right)-k_{2}^{\omega}\left(R_{q}\right)\right)}\right. \text { or } \\
& \prod_{l=k_{1}^{\omega}\left(R_{q}\right)-1}^{k_{2}^{\omega}\left(R_{q}^{1 / \theta}\right)} N_{\omega_{l}} \leqslant \bar{N}^{(1-\varepsilon)\left(k_{2}^{\omega}\left(R_{q}^{1 / \theta}\right)-k_{1}^{\omega}\left(R_{q}\right)\right)} \text { or } \\
& \left.\prod_{l=k_{2}^{\omega}\left(R_{q}^{1 / \theta}\right)+1}^{k_{1}^{\omega}\left(R_{q}^{1 / \theta}\right)} B_{\omega_{l}} \leqslant \bar{B}^{(1-\varepsilon)\left(k_{1}^{\omega}\left(R_{q}^{1 / \theta}\right)-k_{2}^{\omega}\left(R_{q}^{1 / \theta}\right)\right)}\right\} .
\end{aligned}
$$

The last term is bounded above by

$$
\begin{aligned}
& \mathbb{P}\left\{\prod_{l=k_{2}^{\omega}\left(R_{q}\right)+1}^{k_{1}^{\omega}\left(R_{q}\right)} C_{\omega_{l}} \leqslant \bar{C}^{(1-\varepsilon)\left(k_{1}^{\omega}\left(R_{q}\right)-k_{2}^{\omega}\left(R_{q}\right)\right)}\right\} \\
& \quad+\mathbb{P}\left\{\prod_{l=k_{1}^{\omega}\left(R_{q}\right)-1}^{k_{2}^{\omega}\left(R_{q}^{1 / \theta}\right)} N_{\omega_{l}} \leqslant \bar{N}^{(1-\varepsilon)\left(k_{2}^{\omega}\left(R_{q}^{1 / \theta}\right)-k_{1}^{\omega}\left(R_{q}\right)\right)}\right\} \\
& \quad+\mathbb{P}\left\{\prod_{l=k_{2}^{\omega}\left(R_{q}^{1 / \theta}\right)+1}^{k_{1}^{\omega}\left(R_{q}^{1 / \theta}\right)} B_{\omega_{l}} \leqslant \bar{B}^{(1-\varepsilon)\left(k_{1}^{\omega}\left(R_{q}^{1 / \theta}\right)-k_{2}^{\omega}\left(R_{q}^{1 / \theta}\right)\right)}\right\}
\end{aligned}
$$

and by Lemma 4.1 and the union estimate used above each probability is bounded above by $L^{\prime} \gamma^{c^{\prime} q}$ for some constants $L^{\prime}, c^{\prime}>0$, and $\gamma \in(0,1)$. Thus, there exists $q_{0}$ such that each term in the sum is bounded by $1 / 6$ for $q \geqslant q_{0}$ and thus the probability on the left-hand side of (4.20) is bounded below by $1 / 2$ for $q \geqslant q_{0}$.

Denote the event on the left-hand side of (4.20) by $E_{q}$. Observe that the event only depends on the values of $\omega_{i}$ for $i$ satisfying $q=k_{2}^{\omega}\left(R_{q}\right) \leqslant i \leqslant k_{1}^{\omega}\left(R_{q}^{1 / \theta}\right)$ as the latter bound is a stopping time. By virtue of construction, there exists an integer $d \geqslant 1$ such that $k_{1}^{\omega}\left(R_{d^{i} q}^{1 / \theta}\right)<d^{i+1} q$ for all $q$. Therefore, the events $\left\{E_{q}, E_{d q}, E_{d^{2} q}, \ldots\right\}$ are pairwise independent. Further, by the above argument,

$$
\sum_{i=0}^{\infty} \mathbb{P}\left(E_{d^{i} q_{0}}\right) \geqslant \sum_{i=0}^{\infty} 1 / 2=\infty
$$

and so by the Borel-Cantelli lemma $E_{q}$ happens infinitely often. Therefore, adapting the argument involving $s_{c}, s_{n}$, and $s_{b}$ from above, we have proved that for all $\varepsilon^{\prime}>0$ there almost surely exist infinitely many $q \in \mathbb{N}$ such that there exists an approximate $R_{q}$-square $Q$ such that

$$
N\left(Q \cap F_{\omega}, R_{q}^{1 / \theta}\right) \geqslant K R_{q}^{(1-1 / \theta)\left(1-\varepsilon^{\prime}\right) s},
$$


where $s=s_{C}+s_{B}+s_{N}$ is the target lower bound for the spectrum. This completes the proof.

Acknowledgements. This work was started while both authors were resident at the Institut Mittag-Leffler during the 2017 semester programme Fractal Geometry and Dynamics. They are grateful for the stimulating environment. Much of the work was subsequently carried out whilst JMF visited the University of Waterloo in March 2018. He is grateful for the financial support, hospitality, and inspiring research atmosphere. JMF was financially supported by the Leverhulme Trust Research Fellowship RF-2016-500, the EPSRC Standard Grant EP/R015104/1, and the University of Waterloo. ST was financially supported by NSERC Grants 2014-03154 and 2016-03719, and the University of Waterloo.

\section{REFERENCES}

[1] T. Bedford. Crinkly curves, Markov partitions and box dimensions in self-similar sets. PhD Dissertation, University of Warwick, 1984.

[2] K. J. Falconer. Fractal Geometry: Mathematical Foundations and Applications, 2nd edn. John Wiley, Hoboken, NJ, 2003.

[3] J. M. Fraser. Assouad type dimensions and homogeneity of fractals. Trans. Amer. Math. Soc. 366 (2014), 6687-6733.

[4] J. M. Fraser, K. E. Hare, K. G. Hare, S. Troscheit and H. Yu. The Assouad spectrum and the quasi-Assouad dimension: a tale of two spectra. Ann. Acad. Sci. Fenn. Math. 44(1) (2019), 379-387.

[5] J. M. Fraser, J. J. Miao and S. Troscheit. The Assouad dimension of randomly generated fractals. Ergod. Th. \& Dynam. Sys. 38 (2018), 982-1011.

[6] J. M. Fraser and H. Yu. New dimension spectra: finer information on scaling and homogeneity. Adv. Math. 329 (2018), 273-328.

[7] J. M. Fraser and H. Yu. Assouad type spectra for some fractal families. Indiana Univ. Math. J. 67 (2018), 2005-2043.

[8] I. García and K. Hare. Properties of quasi-Assouad dimension. Preprint, 2017, http://arxiv.org/ abs/1703.02526. Ann. Acad. Sci. Fenn., to appear.

[9] D. Gatzouras and S. Lalley. Statistically self-affine sets: Hausdorff and box dimensions. J. Theoret. Probab. 7 (1994), 437-468.

[10] Y. Gui and W. Li. A random version of McMullen-Bedford general Sierpinski carpets and its application. Nonlinearity 21 (2008), 1745-1758.

[11] J. Luukkainen. Assouad dimension: antifractal metrization, porous sets, and homogeneous measures. J. Korean Math. Soc. 35 (1998), 23-76.

[12] J. M. Mackay. Assouad dimension of self-affine carpets. Conform. Geom. Dyn . 15 (2011), 177-187.

[13] C. McMullen. The Hausdorff dimension of general Sierpiński carpets. Nagoya Math. J. 96 (1984), 1-9.

[14] J. C. Robinson. Dimensions, Embeddings, and Attractors (Cambridge Tracts in Mathematics, 186). Cambridge University Press, Cambridge, 2011.

[15] S. Troscheit. The box dimension of random box-like self-affine sets. Indiana Univ. Math. J. 67 (2018), 495-535.

[16] S. Troscheit. The quasi-Assouad dimension of stochastically self-similar sets. Proc. Roy. Soc. Edinburgh Sect. A 150(1) (2020), 261-275. 